\title{
A higher De Ritis ratio (AST/ALT) is a risk factor for progression in high-risk non-muscle invasive bladder cancer
}

\author{
Sachi Fukui-Kawaura1, Takashi Kawahara ${ }^{2}$, Yushi Araki ${ }^{1}$, Reona Nishimura ${ }^{1}$, Koichi \\ Uemura $^{1}$, Kazuhiro Namura ${ }^{1}$, Nobuhiko Mizuno ${ }^{1}$, Masahiro Yao ${ }^{3}$, Hiroji Uemura² \\ and Ichiro Ikeda ${ }^{1}$ \\ ${ }^{1}$ Department of Urology, Yokohama Minami Kyousai Hospital, Yokohama, Japan \\ ${ }^{2}$ Departments of Urology and Renal Transplantation, Yokohama City University Medical Center, Yokohama, Japan \\ ${ }^{3}$ Department of Urology, Yokohama City University, Graduate School of Medicine, Yokohama, Japan \\ Correspondence to: Takashi Kawahara, email: takashi_tk2001@yahoo.co.jp \\ Keywords: AST; ALT; De Ritis; high risk NMIBC \\ Received: October 26, $2020 \quad$ Accepted: March 24, $2021 \quad$ Published: April 27, 2021
}

Copyright: () 2021 Fukui-Kawaura et al. This is an open access article distributed under the terms of the Creative Commons Attribution License (CC BY 3.0), which permits unrestricted use, distribution, and reproduction in any medium, provided the original author and source are credited.

\section{ABSTRACT}

Background: High-risk non-muscle invasive bladder cancer (NMIBC) is thought to be associated with a higher risk of recurrence and progression. A recent study revealed that a high De Ritis ratio was a risk factor in some solid malignancies. This study examined the importance of the De Ritis ratio as a prognostic marker in highrisk NMIBC.

Materials and Methods: A total of 138 patients who were initially diagnosed with high-risk NMIBC between January 2012 to December 2016 were enrolled in this study. The criteria for the high-risk classification followed the EAU guidelines. The recurrence-free and progression-free survival of the higher and lower De Ritis ratio groups were compared. The cut-off value of the De Ritis ratio was set at 1.35, based on a receiver operator curve analysis.

Results: The median observation period was $\mathbf{5 0 . 3}$ months. Among these patients, $32(23.1 \%)$ patients developed recurrent disease and $15(10.9 \%)$ patients showed progression. A multivariate analysis revealed that non-BCG treatment was an independent risk factor for recurrence, and a higher De Ritis ratio was an independent risk factor for cancer progression.

Conclusions: The De Ritis ratio might be a risk factor for progression in high-risk NMIBC.

\section{INTRODUCTION}

Bladder cancer is the eleventh most common malignant disease in the world, and non-muscle invasive bladder cancer (NMIBC) accounts for $75 \%$ of all bladder cancer cases [1]. Although NMIBC is treated by trans-urethral endoscopic surgery, high-risk NMIBC easily progresses to MIBC and shows a high rate of recurrence [2, 3].

The recommended standard treatments for highrisk NMIBC, which is defined by the NCCN and EAU guidelines, include intra-vesicle BCG or repeat TUR-Bt [4]. Although these are considered to be intensive treatments, the rates of recurrence and progression are still high. Thus, more detailed criteria are needed for the risk assessment.
The serum aspartate aminotransaminase (AST)/ alanine aminotransaminase (ALT) ratio was firstly reported by De Ritis in 1957, since then this ratio has been called the De Ritis ratio [5]. ALT is only present in liver cells, while AST is present in heart, renal, brain, muscle, and liver cells [6]. A high De Ritis ratio was reported to be a poor prognostic marker in some solid malignancies [7]. In genitourinary cancer, a high De Ritis ratio was reported to be a poor prognostic marker in prostate, renal, and urothelial carcinoma. In other solid malignancies including breast and lung cancer, a high De Ritis ratio was also reported to be a poor prognostic marker.

This study examined the importance of the De Ritis ratio as a prognostic marker in high-risk NMIBC. 


\section{RESULTS}

The patients' characteristics including age, urinary cytology, pathological T stage, CIS (or not), tumor size, number of tumors, tumor grade, repeat TUR (or not), and BCG instillation are shown in Table 1. The median $($ mean \pm SD) age was 75 years $(74.4 \pm 9.8$ years $)$ and 109 patients $(79.0 \%)$ were male. Higher De Ritis groups showed significantly higher age comparting to the lower De-Ritis group $(p<0.001)$. Among these patients 32 patients (23.2\%) showed recurrence and 15 (10.9\%) showed progression. The median (mean $\pm \mathrm{SD}$ ) observation period was 50.3 months $(51.1 \pm 28.6$ months $)$. The median recurrence-free survival time was 40.8 months (42.7 \pm 28.2 months) and the median progression free survival time was 48.2 months ( $48.2 \pm 29.2$ months). At the time of the initial diagnosis, the rate of high-grade pTa was $25.4 \%$, and the rate of pT1 was $50.0 \%$. Thirtyfour patients $(24.6 \%)$ had CIS, $90(65.2 \%)$ had multiple tumors, and $28(20.3 \%)$ had tumors of $\geq 3 \mathrm{~cm}$ in diameter. Fifty-seven patients $(41.3 \%)$ underwent repeat TUR-Bt and 112 patients $(81.2 \%)$ received postoperative intravesical treatment, including BCG in 101 patients $(73.1 \%)$ and pirarubicin in 11 patients $(8.0 \%)$.

The cut-off value of the De Ritis ratio, which was selected based on the AUROC, was 1.35 (Figure 1A and 1B). Both ROCs for recurrence and progression showed same candidate cut-off points. Kaplan-Meier curve and a log-rank test revealed that the high De Ritis group showed poorer recurrence free and progression free survival ( $p$ $=0.030$ and $p=0.002$, respectively) (Figures 2 and 3). The multivariate analysis revealed that intra-vesicle BCG instillation was independent risk factor for recurrence
( $p=0.002)$. A high De Ritis $(\geq 1.35)$ ratio was the only independent risk factor for progression $(\mathrm{HR}=5.558,95 \%$ $\mathrm{CI}=1.493-20.689, p=0.01)($ Tables 2 and 3$)$.

\section{DISCUSSION}

In this study of patients with NMIBC, the high De Ritis ratio group showed higher rates of recurrence and progression. In the EORTC trial, among patients with high-risk NMIBC, the rate of recurrence within 5 years was approximately $80 \%$, while the rate of progression within 5 years was approximately $50 \%$ [2]. In the CUETO trial, intra-vesicle BCG instillation reduced the rates of recurrence and progression in both intermediate- and highrisk NMIBC [3]. The present study also demonstrated the effectiveness of BCG instillation in reducing recurrence in high-risk NMIBC, although patients with high-risk NMIBC who received BCG treatment showed higher rates of recurrence and progression rate. Thus, the De Ritis ratio might be clue to identifying cases that would benefit from additional treatment, including total cystectomy.

The AST/ALT ratio, which is also known as the De Ritis ratio, was first reported by De Ritis in 1957 [5]. ALT is an enzyme that only exists in hepatic cells, while AST is present in the cells of the liver, kidney, brain, muscle, and other organs [6]. The De Ritis ratio was initially used as a marker of viral hepatitis; however, it is now used as a prognostic marker for various diseases, including hepatic cell carcinoma (HCC), breast cancer, renal cell carcinoma, testicular tumor, and urothelial carcinoma [8-12]. Although the detailed mechanism is still unknown, Li-xian Zhang et al. reported that a higher De Ritis ratio was associated with a poor prognosis in HCC. AST

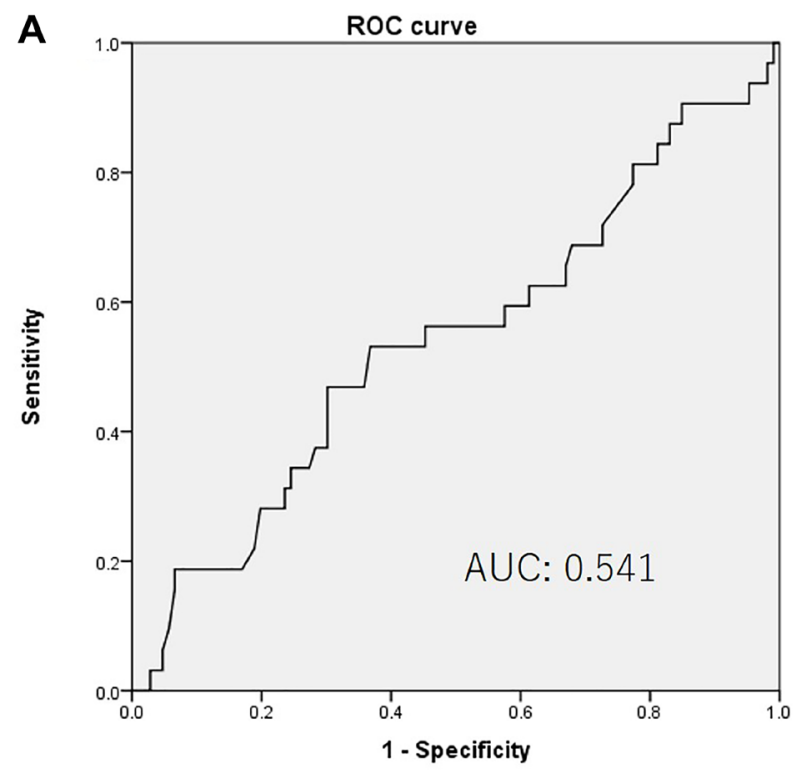

Recurrence
B

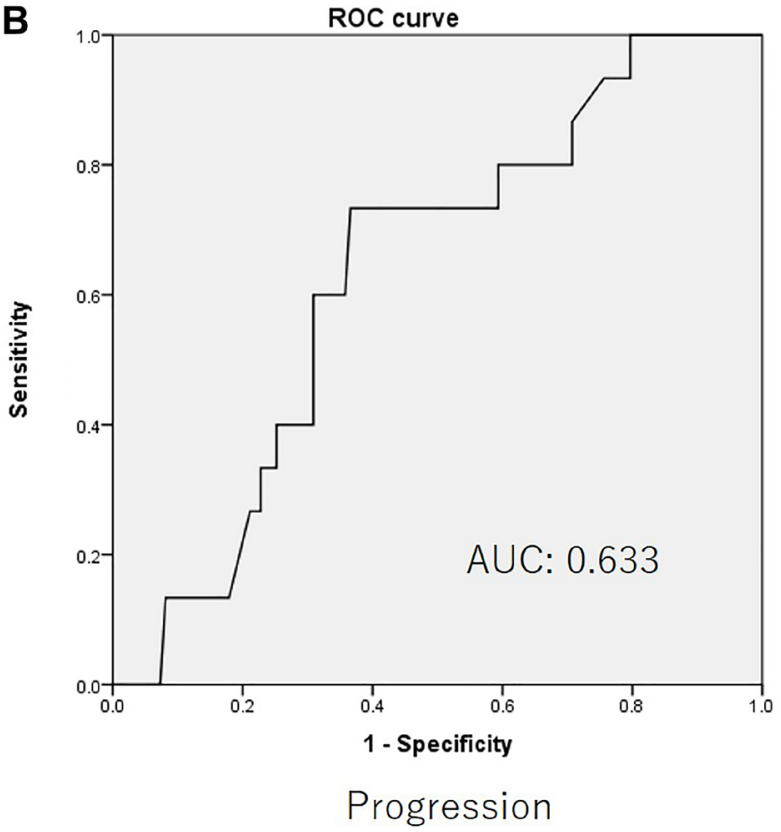

Figure 1: The receiver operator characteristic curve for (A) recurrence and (B) progression. 
Table 1: Patients' characteristics

\begin{tabular}{|c|c|c|c|c|c|}
\hline & & All $(n=138)$ & $\begin{array}{c}\text { AST/ALT }<1.35 \\
(n=81)\end{array}$ & $\begin{array}{c}\text { AST/ALT } \leqq 1.35 \\
(n=57)\end{array}$ & $p$ value \\
\hline \multirow[t]{2}{*}{ Age, yr } & $<75$ & $66(47.8)$ & $50(61.7)$ & $16(28.0)$ & \multirow{2}{*}{0.260} \\
\hline & $75 \leqq$ & $72(52.2)$ & $31(38.3)$ & $41(71.9)$ & \\
\hline \multirow[t]{2}{*}{ Sex } & Female & $29(21.0)$ & $14(17.3)$ & $15(26.3)$ & \multirow{2}{*}{0.201} \\
\hline & Male & $109(79.0)$ & $67(82.7)$ & $42(73.7)$ & \\
\hline \multirow[t]{3}{*}{ Urinary Cytology } & I-III & $50(36.2)$ & $33(40.7)$ & $17(29.8)$ & \multirow{3}{*}{0.308} \\
\hline & IV-V & $79(57.2)$ & $45(55.6)$ & $34(59.6)$ & \\
\hline & Unknouwn & $9(6.5)$ & $3(3.7)$ & $6(10.5)$ & \\
\hline \multirow[t]{4}{*}{$\mathrm{T}$ category } & pT1 & $69(50.0)$ & $41(50.6)$ & $28(49.1)$ & \multirow{4}{*}{0.668} \\
\hline & CIS & $34(24.6)$ & $17(21.0)$ & $17(29.8)$ & \\
\hline & HG pTa & $35(25.4)$ & $23(28.4)$ & $12(21.1)$ & \\
\hline & $\begin{array}{l}\text { Multiple+LG } \\
\text { pTa }+3 \mathrm{~cm} \leqq\end{array}$ & $0(0.0)$ & $0(0.0)$ & $0(0.0)$ & \\
\hline \multirow[t]{2}{*}{ CIS } & No & $104(75.4)$ & $64(79.0)$ & $40(70.2)$ & \multirow{2}{*}{0.237} \\
\hline & Yes & $34(24.6)$ & $17(21.0)$ & $17(29.8)$ & \\
\hline \multirow[t]{3}{*}{ No. of Tumors } & Single & $44(31.9)$ & $29(35.8)$ & $15(26.3)$ & \multirow{3}{*}{0.208} \\
\hline & Multiple & $90(65.2)$ & $49(60.5)$ & $41(71.9)$ & \\
\hline & Unknown & $4(2.9)$ & $3(3.7)$ & $1(1.8)$ & \\
\hline \multirow[t]{2}{*}{ Tumor size } & $<3 \mathrm{~cm}$ & $110(79.7)$ & $64(79.0)$ & $46(80.7)$ & \multirow{2}{*}{0.809} \\
\hline & $3 \mathrm{~cm} \leqq$ & $28(20.3)$ & $17(21.0)$ & $11(19.3)$ & \\
\hline \multirow[t]{3}{*}{ Grade } & Low & $19(13.8)$ & $11(13.6)$ & $8(14.0)$ & \multirow{3}{*}{0.863} \\
\hline & High & $115(83.3)$ & $69(85.1)$ & $46(80.7)$ & \\
\hline & Unknown & $4(2.9)$ & $1(1.2)$ & $3(5.3)$ & \\
\hline \multirow[t]{2}{*}{ second TUR } & No & $81(58.7)$ & $44(54.3)$ & $37(64.9)$ & \multirow{2}{*}{0.215} \\
\hline & Yes & $57(41.3)$ & $37(45.7)$ & $20(35.1)$ & \\
\hline Single instillation & & $103(74.6)$ & $63(77.8)$ & $40(70.2)$ & 0.329 \\
\hline \multicolumn{6}{|c|}{ Additional Delayed Bladder Instillation } \\
\hline & None & $25(18.1)$ & $12(14.8)$ & $13(22.8)$ & \multirow{4}{*}{0.183} \\
\hline & Pirarubicin & $11(8.0)$ & $6(7.4)$ & $5(8.8)$ & \\
\hline & BCG & $101(73.1)$ & $63(77.8)$ & $38(66.7)$ & \\
\hline & unknown & $1(0.7)$ & $0(0.0)$ & $1(1.8)$ & \\
\hline BCG Maintenance & & $11(8.0)$ & $9(11.1)$ & $2(3.5)$ & 0.106 \\
\hline
\end{tabular}

contains mitochondria, while ALT does not. One possible explanation for the association between a higher De Ritis ratio and a poor prognosis in $\mathrm{HCC}$ is that liver dysfunction due to a decrease in mitochondria may increase the serum AST level [8]. Recently Yuk et al. reported that a higher De Ritis ratio was a poor prognostic factor in patients undergoing total cystectomy [13]. The present study is the first to analyze the association between the De Ritis ratio and the prognosis in high-risk NMIBC and found a similar relationship. Our results suggested that for highrisk NMIBC patients with higher De Ritis ratio might consider nao-adjuvant systemic chemotherapy in addition to total cystectomy.
Malignant disease uses the glycolysis system or malateaspartate shuttle as an energy metabolic pathway $[14,15]$. Both AST and ALT carry carbohydrates and are protein metabolic pathways. ALT is involved in the glucose-alanine cycle and AST is a metabolic pathway of the malate-aspartate shuttle in mitochondria [6]. AST also plays an important role in glucose metabolism [6]. Based on these mechanisms, high tumor progression activity may increase tumor metabolism and result in an increase in the AST/ALT ratio.

The present study is associated with several limitations. First, the study was retrospective in nature. Second, the sample size was relatively small. To reveal the usefulness of the De Ritis ratio as a biomarker, a longer- 


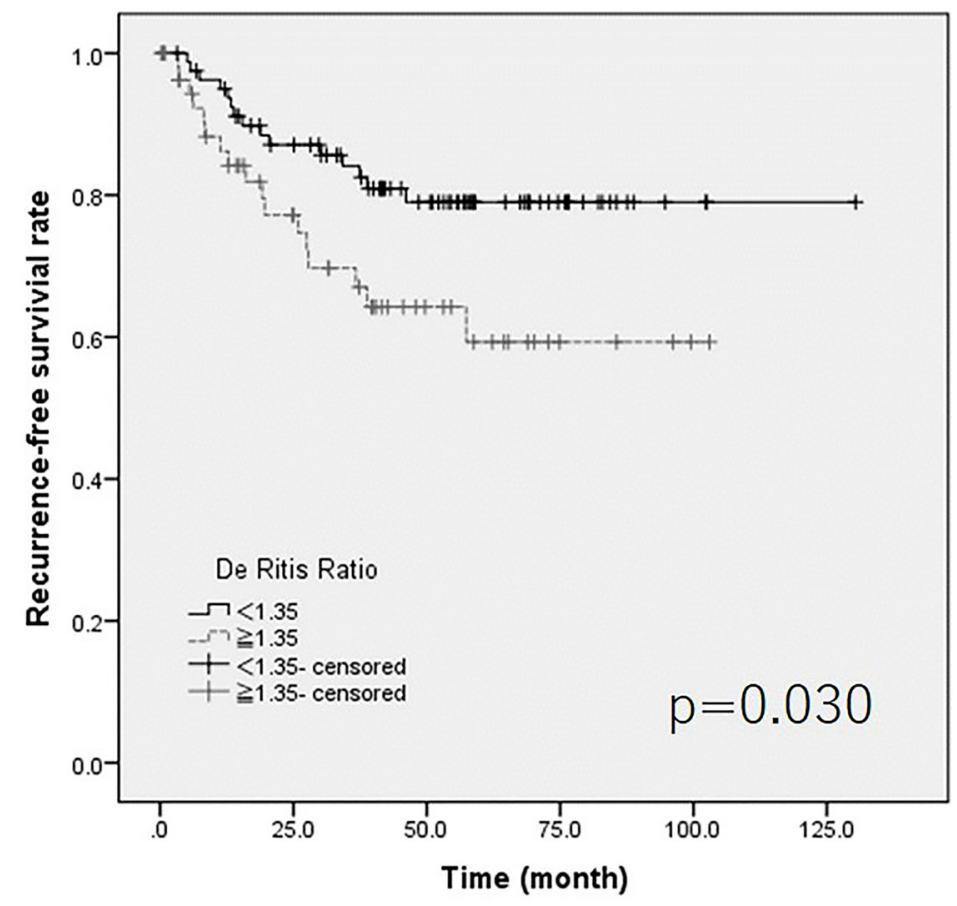

Number at risk

$\begin{array}{llllcll}<1.35 & 80 & 62 & 39 & 14 & 2 & 0 \\ \geqq 1.35 & 56 & 31 & 15 & 4 & 1 & 0\end{array}$

Figure 2: The Kaplan Meier curve for recurrence.

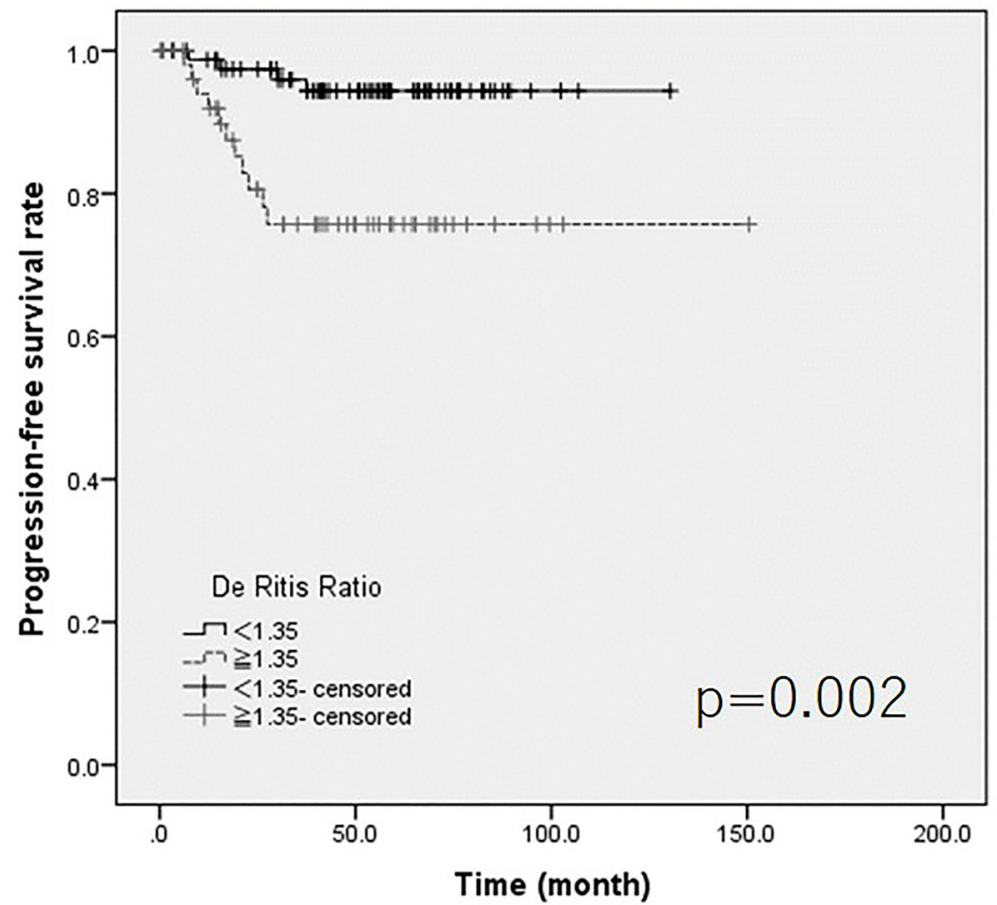

Number at risk

$\begin{array}{llllll}<1.35 & 80 & 47 & 4 & 0 & 0 \\ \geqq 1.35 & 56 & 19 & 2 & 1 & 0\end{array}$

Figure 3: The Kaplan Meier curve for progression. 
Table 2: Multivariate analysis for recurrence

\begin{tabular}{lccccc}
\hline & & HR & CI 95\% & HR & p-value \\
\cline { 5 - 6 } & & & Lower & Upper & 1.198 \\
Age, yr & $<75,75 \leqq$ & 0.512 & 0.219 & 7.532 & 0.123 \\
Sex & Female, Male & 2.369 & 0.745 & 2.871 & 0.144 \\
No. of tumors & Single, Multiple & 1.254 & 0.547 & 3.922 & 0.593 \\
Tumor size & $<3 \mathrm{~cm}, 3 \mathrm{~cm} \leqq$ & 1.626 & 0.674 & 2.487 & 0.279 \\
Single instillation & No, Yes & 1.028 & 0.425 & 0.623 & 0.952 \\
BCG & No, Yes & 0.271 & 0.118 & 5.003 & 0.002 \\
De Ritis Ratio & $<1.35,1.35 \leqq$ & 2.232 & 0.996 & 0.051 \\
\hline
\end{tabular}

Table 3: Multivariate analysis for progression

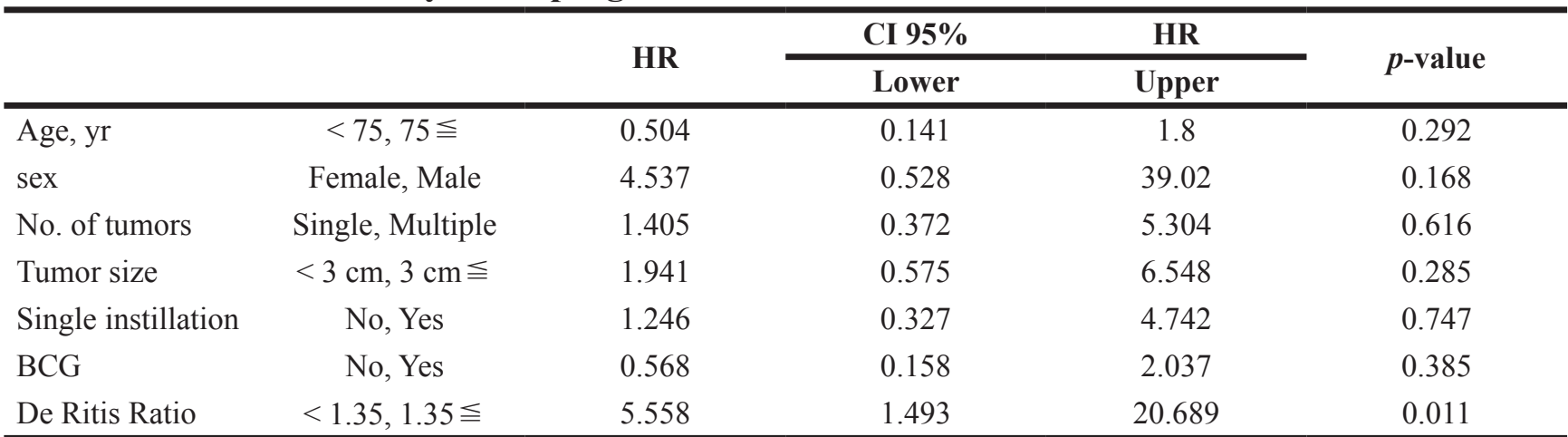

term study of a larger population with a prospective design should be performed. Third, this study showed higher age in higher De Ritis group. No previous study showed the correlation between AST/ALT value and age. Thus, further study is needed.

\section{MATERIALS AND METHODS}

A total of 138 patients who were initially diagnosed with high-risk NMIBC in Yokohama Minami Kyousai Hospital (Yokohama, Japan) between 2012 to 2016 were included in this study. The institutional review board of Yokohama Minami Kyousai Hospital approved this study (IRB No: 1-20-2-63). High-risk NMIBC was defined according to the EAU guidelines as follows: 1) pathological T1, 2) carcinoma in situ (CIS), 3) pathological high-grade urothelial carcinoma, or 3) multiple sites with low-grade and pathological Ta with a maximum diameter of $\geq 3 \mathrm{~cm}$. Some of the patients ( $n=57,41.3 \%$ ) underwent repeat TUR-Bt, while 103 patients $(74.6 \%)$ received 6 courses or more of intravesical chemotherapy using pirarubicin (30 mg in $15 \mathrm{~min}$ ) 10 times per 6 months and intra-vesical BCG $(80 \mathrm{mg}$ in 60 min; Immunobladder, Japan BCG Lab, Tokyo, Japan) once a week. The De Ritis ratio was calculated at the time of preoperative examination. Follow-up examinations included cystoscopy and urinary cytology performed every 3 months until two years from the initial diagnosis.

\section{Statistical analyses}

The patients' characteristics and preoperative factors were analyzed by the Mann-Whitney $U$ test using the SPSS software program (SPSS 22.0, Chicago, IL, USA) and the Graph Pad Prism software program (Graph Pad Software, La Jolla, CA, USA). Candidate cut-off points were identified using the area under receiver operator curve (AUROC). The survival duration was defined as the time between the date of the initial pathological diagnosis and the date of tumor recurrence or progression (muscle invasiveness or metastasis). A log-rank test was performed for comparisons between the high and low De Ritis ratio groups. A Cox regression analysis was performed as a multivariate analysis. $P$ values of $<0.05$ were considered to indicate statistical significance.

\section{CONCLUSIONS}

The De Ritis ratio might be a risk factor for progression in high-risk NMIBC.

\section{Abbreviations}

NMIBC: non-muscle invasive bladder cancer; AST: aspartate aminotransaminase; ALT: alanine aminotransaminase; CIS: carcinoma in situ; HCC: hepatic cell carcinoma; AUROC: area under receiver operator curve. 


\section{Author contributions}

SF, TK wrote the manuscript. YA, RN, KU, KN, NM, MY, HU, II performed the experiment.

\section{Ethics statement}

The institutional review board of Yokohama Minami Kyousai Hospital approved this study (IRB No: 1-202-63) and consent to participate was waivered due to retrospective observational study.

\section{Consent for publication}

The institutional review board of Yokohama Minami Kyousai Hospital approved this study (IRB No: 1-20-263 ) including consent for publication.

\section{Availability of data and materials}

Due to ethical restrictions, the raw data underlying this paper are available upon request from the corresponding author.

\section{CONFLICTS OF INTEREST}

Authors have no conflicts of interest to declare.

\section{REFERENCES}

1. Babjuk M, Burger M, Compérat EM, Gontero P, Mostafid $\mathrm{AH}$, Palou J, van Rhijn BW, Rouprêt M, Shariat SF, Sylvester R, Zigeuner R, Capoun O, Cohen D, et al. European Association of Urology Guidelines on Nonmuscle-invasive Bladder Cancer (TaT1 and Carcinoma In Situ) - 2019 Update. Eur Urol. 2019; 76:639-57. https://doi. org/10.1016/j.eururo.2019.08.016. [PubMed]

2. Sylvester RJ, van der Meijden AP, Oosterlinck W, Witjes JA, Bouffioux C, Denis L, Newling DW, Kurth $\mathrm{K}$. Predicting recurrence and progression in individual patients with stage Ta T1 bladder cancer using EORTC risk tables: a combined analysis of 2596 patients from seven EORTC trials. Eur Urol. 2006; 49:466-465. https://doi. org/10.1016/j.eururo.2005.12.031. [PubMed]

3. Fernandez-Gomez J, Solsona E, Unda M, Martinez-Piñeiro L, Gonzalez M, Hernandez R, Madero R, Ojea A, Pertusa C, Rodriguez-Molina J, Camacho JE, Isorna S, Rabadan M, et al, and Club Urológico Español de Tratamiento Oncológico (CUETO). Prognostic factors in patients with non-muscleinvasive bladder cancer treated with bacillus CalmetteGuerin: multivariate analysis of data from four randomized CUETO trials. Eur Urol. 2008; 53:992-1001. https://doi. org/10.1016/j.eururo.2007.10.006. [PubMed]

4. Babjuk M, Böhle A, Burger M, Capoun O, Cohen D, Compérat EM, Hernández V, Kaasinen E, Palou J, Rouprêt M, van Rhijn
BW, Shariat SF, Soukup V, et al. EAU Guidelines on NonMuscle-invasive Urothelial Carcinoma of the Bladder: Update 2016. Eur Urol. 2017; 71:447-61. https://doi.org/10.1016/j. eururo.2016.05.041. [PubMed]

5. De Ritis F, Coltorti M, Giusti G. An enzymic test for the diagnosis of viral hepatitis; the transaminase serum activities. Clin Chim Acta. 1957; 2:70-4. https://doi. org/10.1016/0009-8981(57)90027-x. [PubMed]

6. Botros M, Sikaris KA. The de ritis ratio: the test of time. Clin Biochem Rev. 2013; 34:117-30. [PubMed]

7. Kang M, Yu J, Sung HH, Jeon HG, Jeong BC, Park SH, Jeon SS, Lee HM, Choi HY, Seo SI. Prognostic impact of the pretreatment aspartate transaminase/alanine transaminase ratio in patients treated with first-line systemic tyrosine kinase inhibitor therapy for metastatic renal cell carcinoma. Int J Urol. 2018; 25:596-603. https://doi.org/10.1111/iju.13574. [PubMed]

8. Zhang LX, Lv Y, Xu AM, Wang HZ. The prognostic significance of serum gamma-glutamyltransferase levels and AST/ALT in primary hepatic carcinoma. BMC Cancer. 2019; 19:841. https://doi.org/10.1186/s12885-019-6011-8. [PubMed]

9. Thornburg JM, Nelson KK, Clem BF, Lane AN, Arumugam S, Simmons A, Eaton JW, Telang S, Chesney J. Targeting aspartate aminotransferase in breast cancer. Breast Cancer Res. 2008; 10:R84. https://doi.org/10.1186/bcr2154. [PubMed]

10. Lee H, Lee SE, Byun SS, Kim HH, Kwak C, Hong SK. De Ritis ratio (aspartate transaminase/alanine transaminase ratio) as a significant prognostic factor after surgical treatment in patients with clear-cell localized renal cell carcinoma: a propensity score-matched study. BJU Int. 2017; 119:261-67. https://doi.org/10.1111/bju.13545. [PubMed]

11. Li Y, Fang D, Bao Z, He A, Guan B, He S, Zhan Y, Gong Y, Li $\mathrm{X}$, Zhou L. High aspartate transaminase/alanine transaminase ratio predicts poor prognosis in patients with localized upper tract urothelial cancer: a propensity score-matched study in a large Chinese center. Onco Targets Ther. 2019; 12:2635-648. https://doi.org/10.2147/OTT.S193771. [PubMed]

12. Gorgel SN, Akin Y, Koc EM, Kose O, Ozcan S, Yilmaz Y. Impact of increased aspartate aminotransferase to alanine aminotransferase (De Ritis) ratio in prognosis of testicular cancer. Investig Clin Urol. 2019; 60:169-75. https://doi. org/10.4111/icu.2019.60.3.169. [PubMed]

13. Yuk HD, Jeong CW, Kwak C, Kim HH, Ku JH. De Ritis Ratio (Aspartate Transaminase/Alanine Transaminase) as a Significant Prognostic Factor in Patients Undergoing Radical Cystectomy with Bladder Urothelial Carcinoma: A Propensity Score-Matched Study. Dis Markers. 2019; 2019:6702964. https://doi.org/10.1155/2019/6702964. [PubMed]

14. Fantin VR, St-Pierre J, Leder P. Attenuation of LDH-A expression uncovers a link between glycolysis, mitochondrial physiology, and tumor maintenance. Cancer Cell. 2006; 9:425-34. https://doi.org/10.1016/j. ccr.2006.04.023. [PubMed]

15. Greenhouse WV, Lehninger AL. Occurrence of the malateaspartate shuttle in various tumor types. Cancer Res. 1976; 36:1392-96. [PubMed] 